\title{
LA LEY DE AMPERE Y SUS DIFICULTADES CONCEPTUALES: UN ANÁLISIS HISTÓRICO EPISTEMOLÓGICO.
}

\author{
AMPERE LAW AND CONCEPTUAL DIFFICULTIES: AN \\ HISTORICAL ANALYSIS EPISTEMOLOGICAL.
}

\author{
Dany Esteban Gallego Quiceno ${ }^{1}$
}

\section{Resumen}

En diferentes investigaciones realizadas acerca de las dificultades conceptuales que se enmarcan dentro de la enseñanza de la ley de Ampere, se ha encontrado que en la mayoría de los textos se deduce la forma integral de la ley de Ampère, para el caso particular de un conductor recto de corriente constante, infinitamente largo, y se generaliza su validez a corrientes "constantes", "estacionarias" o "estables", "encerradas", "envueltas" o "enlazadas" (Kofman, 2000). En muchos casos se pone énfasis en la analogía entre la ley de Ampère para campos magnéticos y la ley de Gauss para campos eléctricos, implicando esto que en muchos textos no se explica que las corrientes deben ser cerradas ni se habla de corrientes cuasiestacionarias.

Estas y otras dificultades conceptuales propician la búsqueda de rutas alternativas para la enseñanza de dicha ley, en ésta investigación se plantea entonces un estudio histórico epistemológico bajo el cual, los estudiantes puedan explicar los fenómenos relacionados por las interacciones entre los campos magnéticos y las corrientes eléctricas, de tal forma que puedan relacionar estos conceptos tanto gráfica, como analíticamente.

Palabras clave: Corriente, Campo, ley de Ampere, Historia, Epistemología.

\section{Abstract}

In different investigations about the conceptual difficulties that are part of the teachin of Ampere's law, has found that most of the texts follows the integral form of Ampere's law for the particular case of a driver constant current straight, infinitely long, and generalize sits validity to current "constant", "stationary" or "stable", "locked up", "wrapped" or "linked" (Kofman, 2000). In many case the emphasis is on the analogy between Ampere's law for magnetic fields and Gauss's law for electric fields, implying that many texts do you explain that the flow should be closed or quasi-stationary current talks.

\footnotetext{
${ }^{1}$ Licenciado en Matemáticas y Física, Docente Facultad de Educación, Universidad de Antioquia, Docente Colegio San José de las Vegas, Colombia. danygallego@yahoo.com
} 


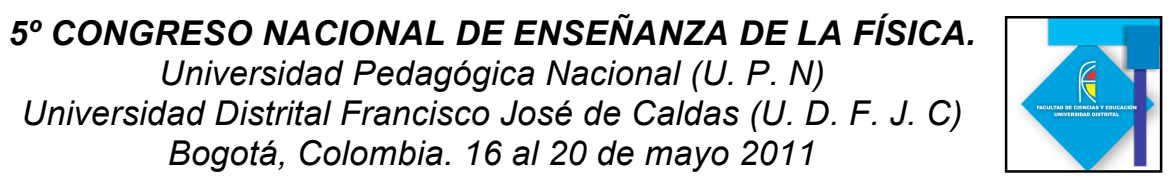

These and other foster conceptual difficulties finding alternative routes to teaching the law, in this research are planning on a historical study epistemological under which students can explain the phenomena related to the interactions between magnetic field sand electric currents, so that they can relate these concepts both graphically, and analytically.

Keywords: Current, Field, Ampere's law, History, Epistemology.

\section{Introducción.}

El presente artículo muestra los resultados de una investigación cualitativa por medio de un estudio intrínseco de casos realizado a 6 estudiantes de nivel universitario, referentes a las diferentes dificultades conceptuales que se enmarcan dentro de los procesos de enseñanza-aprendizaje de la ley de ampere, se encontró que algunas de las dificultades respecto a la ley de Ampere, son fundamentalmente debidos a la manera en que es abordada dicha ley en diferentes textos escolares, se ha encontrado que la ley se deduce de la forma integral, para el caso particular de un conductor recto de corriente constante, infinitamente largo, y se generaliza su validez a corrientes "constantes", "estacionarias" o "estables", "encerradas", "envueltas" o "enlazadas" por la curva $C$ (Kofman, 2000). En varios textos, se pone énfasis en la analogía entre la ley de Ampère para campos magnéticos y la ley de Gauss para campos eléctricos, implicando esto que en pocos textos se explica que las corrientes deben ser cerradas y en ocasiones ni se habla de corrientes cuasiestacionarias.

En la mayoría de los textos indagados durante la investigación, se explica que la aplicabilidad de la ley de Ampère (como la ley de Gauss) se limita a casos de simetría del campo. Un ejemplo particular de este abordaje, se encuentra en el texto de Tipler (1978) en el que el autor discute casos en los que la ley se aplica fácilmente debido a la simetría del campo, y casos en los que la ley no es aplicable (pero sí es válida) por falta de simetría, como los casos de espira y circuito finito que contiene un segmento de corriente. Los problemas a los que se aplica la ley de Ampère, son prácticamente los mismos en todos los textos, incluyendo conductores cilíndricos infinitos, solenoide toroidal y bobina (Kofman, 2000).

Las dificultades conceptuales mostradas en los párrafos anteriores, instan a buscar, rutas conceptuales alternativas, en las que se superen dichas dificultades, debido a que se debe buscar una,

"idea de formación en ciencias que propicie la construcción de modelos de la naturaleza y su puesta en práctica en diferentes escenarios tenga como fundamento una concepción de ciencia que destaca tanto los conceptos y teorías construidos en los campos de la biología, la física y la química, como los procesos, los procedimientos y la dinámica de la elaboración, el contraste y el ajuste de dichos esquemas de conocimiento (Dart,1974).

Se pretende entonces, que los estudiantes puedan explicar los fenómenos relacionados por las interacciones entre los campos magnéticos y las corrientes eléctricas, tomar decisiones argumentadas sobre problemas de su entorno y, en general, ponerlos en práctica en diferentes situaciones, de tal forma que ellos puedan relacionar estos conceptos tanto gráfica, como analíticamente. 
$5^{\circ}$ CONGRESO NACIONAL DE ENSEÑANZA DE LA FÍSICA.

Universidad Pedagógica Nacional (U. P. N)

Universidad Distrital Francisco José de Caldas (U. D. F. J. C)

Bogotá, Colombia. 16 al 20 de mayo 2011

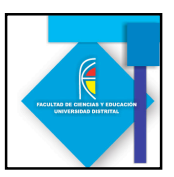

Para mejorar el abordaje conceptual de dicha ley, se propone un análisis de los planteamientos originales de la ley, ya que como se muestra en la investigación de Kofman y Cocarí la ley de Ampere se trata limitadamente en algunos de los textos más utilizados en la formación universitaria. De otro lado es fundamental comprender que la complejidad matemática que tiene la demostración de la ley de Ampere a partir de la ley de Biot Savart no es razón para enseñar estas leyes de forma simplificada y muchos menos abordarla tal y como se aborda en los textos universitarios.

Se hace entonces necesario buscar herramientas de enseñanza, que permitan un mejor abordaje menos simplista y más conceptual.

\section{La ley de ampere en los procesos de enseñanza-aprendizaje.}

Según los estándares en ciencias propuestos por el ministerio de educación nacional en Colombia,

"la dinámica del mundo contemporáneo exige a cualquier persona que viva y conviva en él, tener una formación básica en ciencias naturales. Por medio de ésta los estudiantes deben tener acceso a los procedimientos e ideas centrales de la ciencia, de tal forma que se les permita entender y relacionar elementos de su cotidianidad $y$, por ende, desenvolverse de una manera más significativa en ella"².

En el caso de las interacciones entre un campo magnético y de una corriente eléctrica, el estudiante debe estar en capacidad de describir y relacionar los conceptos asociados a dichos fenómenos, representarlos y expresar las relaciones que ligan unas y otras variables.

\section{Como lo expresan Kofman y Concarí:}

"La ley de Ampère es enseñada en los cursos básicos de física en la universidad como una de las leyes fundamentales del electromagnetismo. En su forma integral, la misma es un instrumento que simplifica la evaluación del campo magnético producido por distribuciones de corriente que presentan simetrías con módulo constante en las líneas de campo. Se lograron identificar algunos problemas relativos a la aplicación de esta ley, en lo que hace a la identificación del circuito de corriente que origina el campo magnético. Algo similar ha ocurrido con relación al cálculo de campos y flujos magnéticos creados por corrientes variables, situaciones que generaban dudas a los alumnos en cuanto al significado de corrientes estacionarias o cuasi estacionarias que se establecen como condiciones de validez de esa ley. Ninguno de estos dos aspectos está claramente explicitado en la bibliografía de los cursos básicos de electromagnetismo" (Kofman, H. Y Concari. 2000).

Por lo anterior manifestado, las indagaciones de carácter educativo no pueden ser ajenas a las dificultades de los estudiantes para representar eventos asociados a las interacciones entre los campos magnéticos y la corriente eléctrica; si bien este un

\footnotetext{
${ }^{2}$ Estándares curriculares para ciencias naturales y educación ambiental, Ministerio de Educación Nacional. www.mineducacion.gov.co Febrero de 2010.
} 


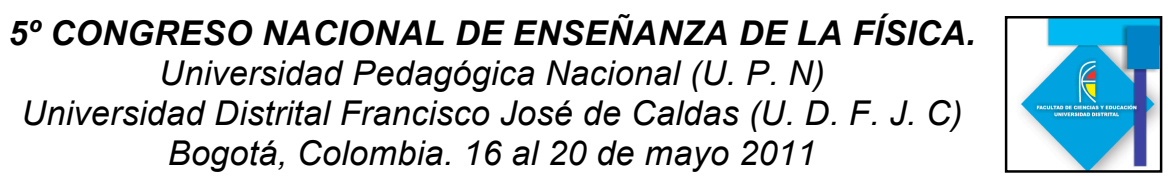

fenómeno comúnmente observado en nuestro quehacer diario, su representación física es limitada, situación que restringe la adecuada resolución de problemas.

Por tanto, se hace necesario reconocer las dificultades conceptuales de los estudiantes, modelos mentales, descripción física y representación gráfica de la situación planteada para así implementar actividades experimentales y estructuras teóricas que contribuyan en la elaboración de modelos más consistentes con el fenómeno en cuestión; desde esta investigación se propone un análisis de los planteamientos originales, para que de este modo se contribuya con una mejor comprensión del fenómeno físico.

\section{Propuesta de enseñanza de la Ley de Ampere.}

Cuando se expresa que la ley de Ampére explica, que:

"la circulación de la intensidad del campo magnético en un contorno cerrado es igual a la corriente que lo recorre en ese contorno, en donde el campo magnético es un campo vectorial con forma circular, cuyas líneas encierran la corriente y la dirección del campo en un punto es tangencial al círculo que encierra la corriente, teniendo en cuenta que el campo magnético disminuye inversamente con la distancia al conductor (Popovic, 2001),

Se dejan de lado relaciones fundamentales y aprehensibles para los estudiantes, por un lado se muestra la limitación que contienen diferentes textos de la ley de Ampere, y por otro las dificultades conceptuales que acarrean un tratamiento tan poco conceptual de la misma, a su vez se sostiene que

"la complejidad matemática que representa la demostración de la ley de Ampère a partir de la ley de Biot y Savart para el caso general de una espira cerrada con corriente, no justificaría la forma simplificada con que se la está enseñando. En todo caso, lo más correcto sería formular el planteo general, mostrando su resultado, y dejar la demostración matemática para un eventual curso más avanzado" Kofman, H. Y Concari. 2000).

Como solución alternativa a los procesos de enseñanza aprendizaje de ésta temática fundamental en los cursos de física, se plantea el uso de textos originales y de interacciones cotidianas donde se pueda poner en evidencia cuales son las corrientes que originan el campo magnético que interviene en la ley de Ampère. De manera similar, sin mayores detalles matemáticos, se podría incluir el estudio del grado de validez de la ley para corrientes cuasiestacionarias.

\section{Resultados de la Intervención.}

La propuesta de enseñanza-aprendizaje de la ley de ampere, basada en un análisis histórico epistemológico permitió a los 6 casos participantes en la investigación, superar las dificultades conceptuales que presentaban al inicio de la intervención, comprendieron que la ley necesariamente no se deduce de la forma integral, para el caso particular de un conductor recto de corriente constante, infinitamente largo, y se generaliza su validez a corrientes "constantes", "estacionarias" o "estables", 

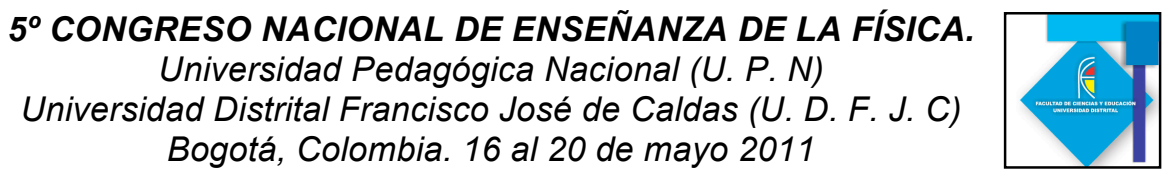

"encerradas", "envueltas" o "enlazadas". No se hizo énfasis en la analogía entre la ley de Ampère para campos magnéticos y la ley de Gauss para campos eléctricos, lo cual permitió comprender que las corrientes deben ser cerradas y que el fenómeno también puede ser estudiado en corrientes cuasiestacionarias.

\section{Referencias Bibliográficas}

Alexander, P. A. Y Kulikowich, J. M. (1994). Learning from physics text: a sinthesis of recent research. Journal of Research in Science Teaching. 31 (9): 895-911.

Dart, F. (1974) Electricidad. Editorial Labor S.A. Barcelona. pp. 9-15

Gallego, D. Aguilar, Y.(2009) La Historia y la Epistemología de la Física como una tentativa para organizar y recontextualizar fenómenos físicos, En Memorias: I Congreso Nacional de Maestros en Formación en enseñanza de la Física. Ponencia. Medellín.

Estándares curriculares para ciencias naturales y educación ambiental, Ministerio de Educación Nacional. www.mineducacion.gov.co Febrero de 2011.

Kofman, H. Concari. (2000) Dificultades conceptuales con la ley de ampère: análisis bibliográfico y simulación como propuesta. Actas del V Simposio de Investigadores en Educación en Física. Argentina.

Popovic Zoya. Introducción al electromagnetismo. $1^{\text {a }}$ ed. México: Continental. 2001

Pérez, M. Varela, P. Orígenes del electromagnetismo. Oersted y Ampere. En Revista Eureka sobre Enseñanza y Divulgación de las Ciencias (2005), Vol. 2, No 1, pp. 118119.

TIPLER, P. A. (1978). Física. Tomo II. Reverté. España. 